\title{
Placental IL-10 Dysregulation and Association With Bronchopulmonary Dysplasia Risk
}

\author{
ELISABETH C. McGOWAN, STEFAN KOSTADINOV, KATHRYN McLEAN, FRANCESCA GOTSCH, DEBORAH VENTURINI, \\ ROBERTO ROMERO, ABBOT R. LAPTOOK, AND SURENDRA SHARMA
}

\begin{abstract}
Departments of Pediatrics [E.C.M., K.M., D.V., A.R.L., S.S.] and Pathology [S.K.], Women and Infants Hospital, Warren Alpert Medical School of Brown University, Providence, Rhode Island 02915; Perinatology Research Branch [F.G., R.R.], Eunice Kennedy Shriver National Institute of Child Health and Human Development (NICHD), NIH/DHHS, Detroit, Michigan 48210
\end{abstract}

\begin{abstract}
Cytokine profiles in amniotic fluid, cord serum, and tracheal aspirate of premature infants suggest a shift toward a proinflammatory state. Cytokines also contribute to the pathogenesis of bronchopulmonary dysplasia (BPD). We hypothesize that the initiating events for BPD are reflected in the placenta and propose that placental expression of cytokines provide a blueprint of events leading to BPD. This is a retrospective, case-controlled study of placental cytokines of premature infants with $(n=49)$ and without ( $n=49)$ BPD, matched for gender, birth weight, and year of birth at Women and Infants Hospital between 2003 and 2005. Cytokine expression, including IL-6 and IL-10, was determined by immunohistochemistry in membrane rolls, umbilical cords, and placentas. IL-6 was similarly expressed in all tissues of infants with and without BPD. In contrast, anti-inflammatory cytokine IL-10 was less prominent in the placenta of patients with BPD compared with those without BPD. IL-10 expression in the villous trophoblast layer was associated with a reduced odds ratio of developing BPD (adjusted OR $0.08,95 \%$ CI $0.01-0.70, p=0.02$ ). These results suggest that a placental balance between inflammatory and anti-inflammatory cytokines is crucial to normal lung organogenesis. Importantly, IL-10 seems to be protective against the development of BPD. (Pediatr Res 66: 455-460, 2009)
\end{abstract}

$\mathrm{B}$ ronchopulmonary dysplasia (BPD) is the most common major morbidity of prematurity, and its frequency is higher with decreasing birth weight (BW) and gestational age (GA). BPD is associated with longer neonatal hospitalizations, neurodevelopmental impairment, and high rates of rehospitalization within the first $2 \mathrm{y}$ of life (1-4). Despite improved survival of premature infants over the past $15 \mathrm{y}$, the incidence of BPD remains unchanged among infants with a $B W<1250 \mathrm{~g}$, ranging between 12 and $62 \%$ (5).

The pathology of BPD has shifted from airway injury, inflammation, and parenchymal fibrosis (6) to a new BPD, characterized by an impairment of alveolar and vascular development (7). The pathogenesis of this new BPD is multifactorial, with environmental contributions from intrauterine infection, inflammation, and cytokine exposure as well as genetic heritability $(8,9)$. Supportive data linking infection/

Received January 21, 2009; accepted May 28, 2009.

Correspondence: Surendra Sharma, M.D., Ph.D., Department of Pediatrics, Women and Infants Hospital, 101 Dudley Street, Providence, RI 02905; e-mail: ssharma@wihri.org

Supported in part by the grant from NIH NCRR (P20RR018728), the Eunice Kennedy Shriver National Institute of Child Health and Human Development, NIH, DHHS, and a subcontract WSU05056 under NICHD contract N01-HD-2-3342. inflammation to BPD include the higher rates of histologic chorioamnionitis among infants with BPD compared with those without BPD $(10,11)$. Several proinflammatory cytokines have been linked to BPD. In particular, elevated concentrations of IL-6 in umbilical cord blood (12), amniotic fluid $(13,14)$, and tracheal aspirates (15) have been documented in preterm infants with BPD. In contrast, reduced levels of IL-10, a cytokine with potent antiinflammatory properties, have been reported in tracheal aspirates of patients with BPD $(16,17)$. It has been suggested that the susceptibility of preterm infants to BPD may in part reflect an inability to regulate inflammation through the decreased expression of IL-10 (18).

There has been little investigation of cytokine expression within the placenta and the potential association with BPD. The presence or absence of placental inflammation in maternal and fetal compartments can potentially provide further insight in understanding the pathogenesis of BPD. These changes in specific placental compartments may be linked to subsequent local organ inflammatory response and thereby serve as a blueprint to trigger events predisposing to the development of BPD. We specifically hypothesize that BPD among infants with a $\mathrm{BW}<1250 \mathrm{~g}$ is associated with an imbalance between expression of "proinflammatory" and "anti-inflammatory" cytokines in specific compartments of the placenta.

\section{METHODS}

This study was approved by the Institutional Review Board of Women and Infants' Hospital of Rhode Island (WIHRI). A waiver of consent was granted to perform this study.

Preliminary studies. Archived placental tissue of 10 infants, born between 24 and 26 wk of gestation, was used to determine the adequacy of immunohistochemistry (IHC) staining of paraffin-embedded tissue for IL-6, IL-8, TNF- $\alpha$, and IL-10. An additional 19 placentas were stained to facilitate determination of sample size.

Study. This was a retrospective, case-controlled study. Infants included were $\leq 1250 \mathrm{~g}$, born at WIHRI between January 2003 and June 2005, and whose placental tissue had been collected at delivery. Pairs of infants with and without BPD were matched for gender, BW, and birth within 6 mo of each other. Exclusion criteria were major congenital anomalies and death before 36-wk postmenstrual age (PMA). The NICU admission log was used to capture eligible infants.

Formalin-fixed, paraffin-embedded placental tissue blocks were retrieved from pathology archives. Sections were cut $(5-\mu \mathrm{m}$ thick) to provide the

Abbreviations: BPD, bronchopulmonary dysplasia; IHC, immunohistochemistry 
extraplacental membrane roll (for examination of the amnion and chorion), umbilical cord (for examination of the vein, artery, and stroma), and fullthickness placental disc (for examination of the amnion, chorion, decidua, villous trophoblast layer and stroma, and extravillous trophoblast).

Immunohistochemistry. Antigen retrieval of deparaffinized sections was performed using a citric acid based unmasking solution (Vector, Burlingame, $\mathrm{CA}$ ) and microwave oven technique. Sections were blocked for $1 \mathrm{~h}$ with $3 \%$ goat serum and incubated with the respective primary polyclonal rabbit antibody (1:100, Research and Diagnostic Systems, Inc.) in a humidified chamber for $1 \mathrm{~h}$ in room air, followed by biotinylated secondary antirabbit antibody (VectaStain Elite Kit) for 45 min. Immunolabeling was performed by a standard Avidin-Biotin ( $\mathrm{ABC}$ ) technique. Labeling was developed with 0.05\% 3,3'diaminobenzidine (DAB; Sigma Chemical Co., St. Louis, MO), and slides were counter stained with hematoxylin (Fisher Scientific, Kalamazoo, MI).

Positive controls were previously stained tissues that showed moderate to severe expression of the corresponding cytokine. Negative controls were standard rabbit IgG controls. Every batch of staining included seven cases and their matched controls, as well as two positive and two negative controls.

Grading was done by two independent, blinded reviewers (SK and EM), with supervision by the third reviewer (SS). A categorical grading system was used, 0 for no staining, 1 for mild staining, and 2 for intense staining. Because of potential grading subjectivity, interobserver variability was assessed (see data analysis).

Clinical data. Clinical data were collected by chart review using predefined variables. Maternal data included demographics, and obstetric variables including antenatal steroid use, antibiotic use, diabetes, hypertension (chronic versus pregnancy induced), prolonged rupture of membranes $(>18$ h), preeclampsia, preterm labor, preterm premature rupture of membranes (PPROM), clinical chorioamnionitis (maternal temperature $\geq 37.8^{\circ} \mathrm{C}$ and uterine tenderness \pm malodorous vaginal discharge and maternal white blood cell count $>15,000$ ), histologic chorioamnionitis (any grade of inflammation documented on the placental report retrieved from pathology archives), and mode of delivery.

Neonatal data included BW, GA (based on Obstetric best estimate), race, gender, and delivery room data. BPD was defined by the use of supplemental oxygen to maintain oxygen saturation $>90 \%$ at 36-wk PMA (19). Infant morbidities included number of days on ventilator support, air leaks, surfactant administration, late-onset sepsis (LOS, culture positive), necrotizing enterocolitis (NEC, $\geq$ Bell's Stage II), patent ductus arteriosus (PDA, treated with indomethacin or ligation), intraventricular hemorrhage (IVH, $\geq$ grade 3 ), cystic periventricular leukomalacia (PVL), and retinopathy of prematurity (ROP, $\geq$ stage III).

Sample size and data analysis. Based on our preliminary studies of trends in differences of IL-6 staining in placental compartments, a sample size of 64 patients (32 in each group) was needed to provide $80 \%$ power to reject the null hypothesis with a two-sided $\alpha$-level of 0.05 . In our study period, we analyzed 49 cases (with BPD) and 49 controls (without BPD).

Interobserver agreement for grading of cytokine expression was analyzed using kappa values derived from the assessment of 11 different placental compartments. Group comparisons for maternal and infant data were analyzed by $\chi^{2}$ for dichotomous variables and $t$ test for continuous variables. Associations between cytokine expression (in each placental compartment) and BPD were initially performed by univariate analysis using $\chi^{2}$. The latter analysis was done using data in matched pairs, instead of composite groups. To simplify the statistical analysis of the relationship between cytokines and BPD, we collapsed grades 1 and 2 staining into "any" staining. Therefore, grading was grouped as "none" (grade 0) versus "any" (grade 1 or 2). Conditional logistic regression was used on the matched data to determine associations between placental expression of cytokines and BPD and was adjusted for GA and race. Results are expressed as an OR with 95\% CI. A $p$ value of $<0.05$ was considered statistically significant.

\section{RESULTS}

Our pilot experiments established the conditions for IHC analysis. Staining of the placental tissue of 29 infants indicated consistent staining for IL-6 and IL-10 but was not observed for TNF- $\alpha$ and IL-8. Based on these results, staining for the main study was limited to IL-6 and IL-10 to facilitate a larger number of placental tissue block assessments. Grading of staining agreement between the two raters varied from kappa values of 0.71 to 0.92 with an overall kappa of 0.82 .
Table 1. Maternal data

\begin{tabular}{lcc}
\hline & $\begin{array}{c}\text { BPD } \\
(n=47)(\%)^{*}\end{array}$ & $\begin{array}{c}\text { No BPD } \\
(n=49)(\%)\end{array}$ \\
\hline Hypertension & 13 & 24 \\
Preeclampsia & 6 & 16 \\
PPROM & 34 & 26 \\
Prolonged rupture of membranes & 32 & 22 \\
Diabetes & 4 & 6 \\
Abruption & 19 & 20 \\
Chorioamnionitis & & \\
$\quad$ Clinical & 21 & 12 \\
$\quad$ Histological & 51 & 57 \\
Steroids (complete course) & 62 & 58 \\
Antibiotics (any prior to delivery) & 75 & 67 \\
Spontaneous labor & 68 & 65 \\
Cesarean delivery & 62 & 71 \\
\hline
\end{tabular}

* Two sets of twins in this group.

Table 2. Infant demographics and delivery room data

\begin{tabular}{lcc}
\hline & $\begin{array}{c}\text { BPD } \\
(n=49)\end{array}$ & $\begin{array}{c}\text { No BPD } \\
(n=49)\end{array}$ \\
\hline Birth weight* $(\mathrm{g})$ & $814 \pm 172$ & $879 \pm 208$ \\
Male & $55 \%$ & $55 \%$ \\
Gestational age* $\dagger$ & $25.9 \pm 1.5$ & $26.7 \pm 1.8$ \\
Race & & \\
Caucasian & $80 \%$ & $60 \%$ \\
Hispanic & $10 \%$ & $22 \%$ \\
African American & $4 \%$ & $14 \%$ \\
Other & $6 \%$ & $4 \%$ \\
Small for gestational age & $10 \%$ & $14 \%$ \\
Intubated & $94 \%$ & $85 \%$ \\
Chest compressions & $0 \%$ & $2 \%$ \\
\hline
\end{tabular}

$*$ Mean $\pm \mathrm{SD}$.

$\dagger p=0.03$.

$\ddagger p=0.07$.

There were 348 in-born patients, with a BW $\leq 1250$ g born between January 2003 and June 2005 with available placental tissue. Deaths occurred in 34 patients before 36 wk PMA, leaving 314 patients (106 with BPD and 208 without BPD) available for this study. Using matching criteria, 98 infants were included in this study: 49 with BPD and 49 without BPD.

Medical complications of pregnancy (diabetes, hypertension, preeclampsia) were similar between the groups (see Table 1). There was no difference in the percentage of women with PPROM, prolonged rupture of membranes, or histologic and clinical chorioamnionitis (CA). The incidence of histologic CA was more than two times greater than clinical CA.

Infants were matched for BW, gender, and interval of birth (see Table 2). Despite the matching criteria, there was a small difference in GA; infants with BPD were almost 1 wk less mature. There was a trend for a difference in distribution of race with a greater predominance of Caucasian patients, and less African Americans and Hispanic infants with BPD. Delivery room interventions were similar between groups. There was a difference between groups receiving surfactant (Table 3) but this was not felt to be clinically important. As expected, infants with BPD spent more days on ventilators and had more frequent postnatal steroid use. Morbidities that were higher in infants with BPD were PDA's requiring treatment and ROP $\geq$ stage III. 
Table 3. Infant morbidities

\begin{tabular}{lccc}
\hline & $\begin{array}{c}\text { BPD } \\
(n=49)\end{array}$ & $\begin{array}{c}\text { No BPD } \\
(n=49)\end{array}$ & $p$ \\
\hline Surfactant use & $100 \%$ & $90 \%$ & 0.02 \\
Air leak & $20 \%$ & $10 \%$ & $\mathrm{NS}$ \\
IVH ( $\geq$ grade 3) & $8 \%$ & $6 \%$ & $\mathrm{NS}$ \\
PDA treatment (indomethacin & $49 \%$ & $14 \%$ & 0.0002 \\
$\quad$ or ligation) & & & \\
Late-onset sepsis (culture & $33 \%$ & $25 \%$ & $\mathrm{NS}$ \\
$\quad$ positive) & $6 \%$ & $10 \%$ & $\mathrm{NS}$ \\
NEC & $10 \%$ & $6 \%$ & $\mathrm{NS}$ \\
PVL & $32 \%$ & $13(0-55 \mathrm{~d})$ & 0.001 \\
Days on ventilator & $(5-109 \mathrm{~d}) *$ & 0.003 \\
Postnatal steroid use & $33 \%$ & $8 \%$ & 0.02 \\
ROP ( $\geq$ stage III) & & & \\
\hline
\end{tabular}

* Median (range).

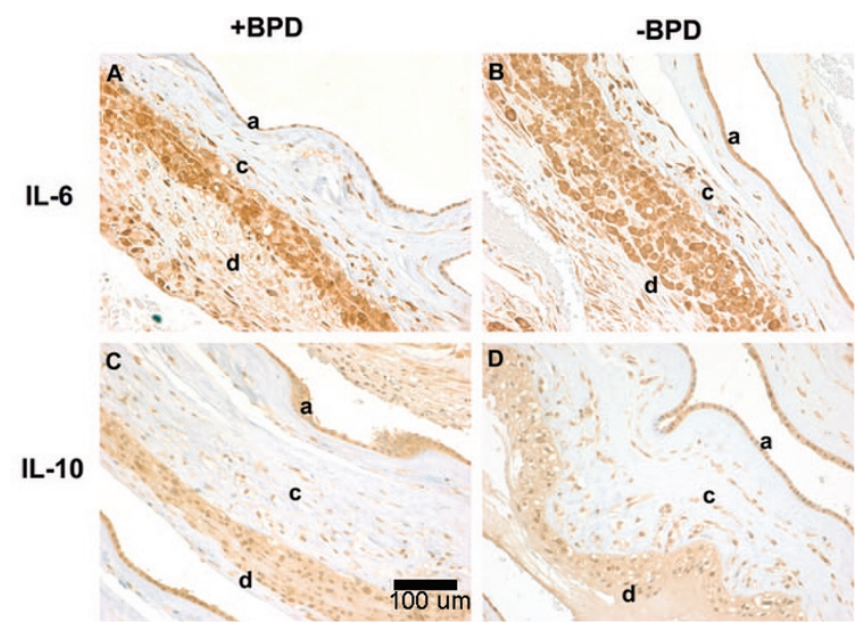

Figure 1. Representative immunostaining of IL- 6 and IL-10 in extraplacental membrane rolls from patients with and without BPD, magnification $\times 20$. $A$ : Grade 2 (intense) staining of IL-6 is seen in amnion (a), chorion (c), and decidua (d) of the extraplacental membrane roll from patients with BPD. $B$ : Grade 2 staining of IL-6 is seen in the amnion, chorion, and decidua of the extraplacental membrane roll of patients without BPD. C: Grade 1 (mild) staining of IL-10 is seen in the amnion, chorion, and decidua of the extraplacental membrane roll from patients with BPD. $D$ : Grade 1 staining of IL-10 seen in the amnion, chorion, and decidua of the extraplacental membrane roll of patients without BPD.

IHC analysis of IL-6. IL-6 was widely expressed in all placental compartments. In our extraplacental membrane rolls, IL-6 was present in both amnion and chorion, and patterns were similar between newborns with and without BPD (Fig. $1 A$ and $B)$. In patients with BPD, 82\% (36/44) showed significant staining (grade 1 or 2) in the amnion and 90\% (43/48) had positive staining in the chorion. For patients without BPD, there was $68 \%$ (30/44) and 88\% (39/48) positive staining for IL-6 in the amnion and chorion, respectively.

IL-6 was also widely expressed in the villous trophoblasts (Fig. $2 A$ and $B$ ). The distribution of trophoblast staining for patients with BPD was as follows: grade 0 $(4 \%)$, grade $1(55 \%)$, and grade $2(40 \%)$. This distribution pattern was similar for villous trophoblasts of patients without BPD as well.

IL-6 staining in the umbilical cord vessels was not robust. In patients with BPD, only $27 \%$ (13/48) showed staining in the arterial endothelium and $31 \%(15 / 48)$ in the venous endothe-

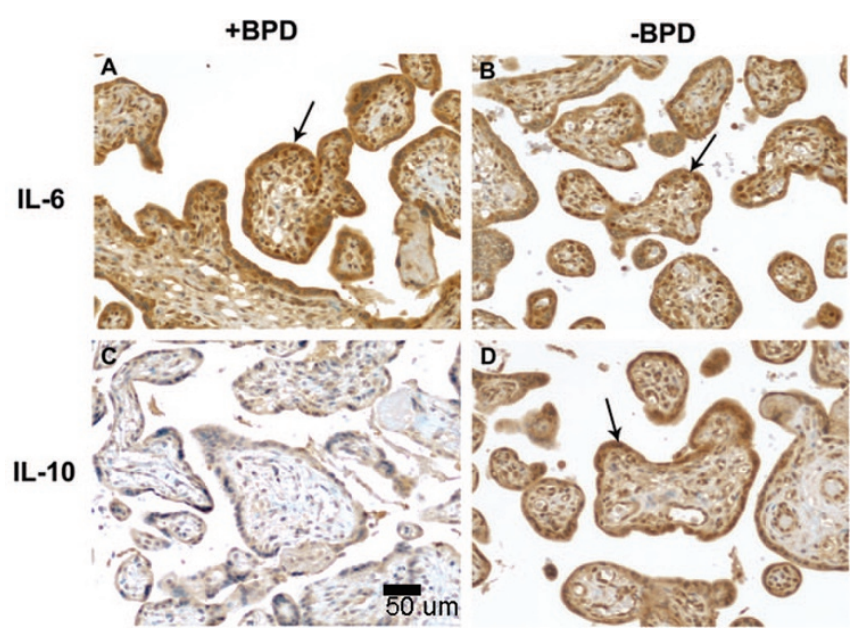

Figure 2. Representative immunohistochemical analysis of IL-6 and IL-10 in placental tissue from patients with or without BPD, magnification $\times 20$. A: Grade 2 (intense) staining of IL-6 is seen in the placental villous trophoblasts in patients with BPD. $B$ : Grade 2 staining of IL- 6 is seen in the placental villous trophoblasts in patients without BPD. $C$ : Grade 0 (no staining) of IL-10 is seen in the villous trophoblasts in patients with BPD. D: Grade 2 staining of IL-10 is seen in the villous trophoblasts in patients without BPD.

lium, and the staining was predominantly mild (grade 1). This pattern was also similar for infants without BPD. There was more abundant staining in the cord stroma, with $75 \%$ of patients both with and without BPD demonstrating staining for IL-6 (data not shown).

IHC analysis of IL-10. Unlike IL-6, the overall staining for IL-10 was comparatively less prominent in all the placental compartments surveyed. For example, IL-10 staining was less intense in the extraplacental membrane roll (Fig. $1 C$ and $D$ ). In the amnion of patients with BPD $28 \%(10 / 36)$ had no staining, while $64 \%(23 / 36)$ had grade 1 staining and the remaining $8 \%(3 / 56)$ had grade 2 . This distribution was similar for the chorion as well. A similar pattern of staining was present for infants without BPD. Interestingly, the adjoining decidual area showed no staining for IL-10. The umbilical cord tissue also showed little staining for IL-10, irrespective of the presence or absence of BPD.

There were differences in the staining for IL-10 in placental villous trophoblasts between patients with and without BPD (Fig. $2 C$ and $D$ ). Sixty percent $(23 / 38)$ of the patients with BPD had no staining, while only $40 \%(15 / 38)$ had grade 1 staining. In contrast, for those patients without BPD, $63 \%$ (21/38) had grade 1 or 2 IL-10 staining.

Associations between cytokines and BPD. There were no associations between IL-6 and BPD for all compartments examined. Similarly, there were no associations between IL-10 expression in the cord or membrane staining and BPD. However, as illustrated in Figure 2 and quantified in Figure 3, IL-10 expression in the trophoblast and stroma differed among infants with and without BPD. Specifically, trophoblast IL-10 expression was associated with a reduction in the odds of developing BPD (OR 0.08, 95\% CI 0.01-0.64, $p=0.017$ ). IL-10 expression in the villous stroma was also associated with a decreased risk of BPD (OR 0.25, CI 0.07-0.89, $p=$ 0.03 ). Adjustment for race and GA did not change this rela- 


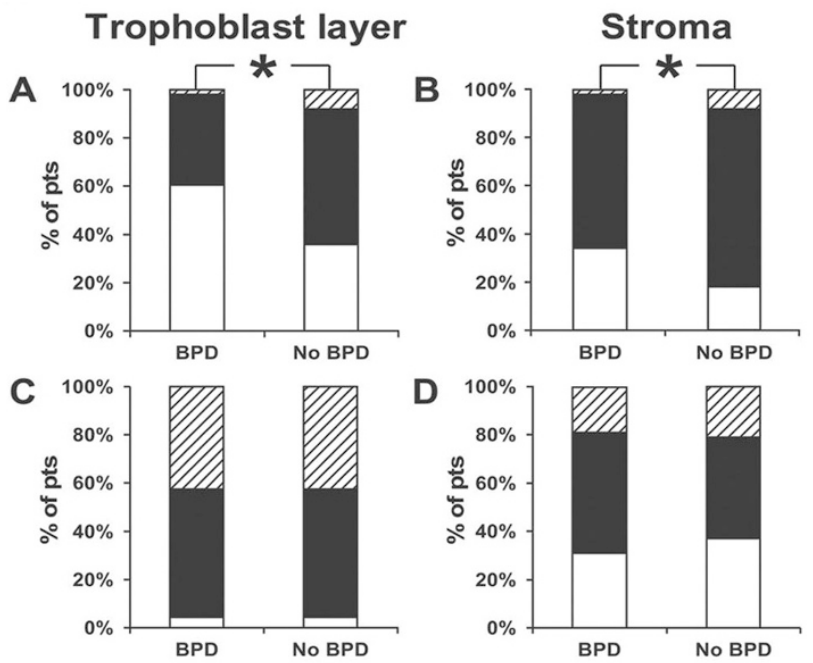

Figure 3. Grade distribution expressed as percent of patients (pts). Grade 0 white bar, grade 1 black bar, and grade 3 shaded bar of immunostaining in the placenta. $(A, B)$ The expression of IL-10 in patients without BPD is associated with a reduction in the odds of developing BPD in adjusted analyses for the villous trophoblast layer (OR 0.08 , CI $0.01-0.70 ; p=0.02$ ) and stroma (OR 0.12, CI 0.02-0.60, $p=0.01)$. $(C, D)$ A similar distribution of IL-6 staining grades seen in the trophoblast layer and stroma among infants with and without BPD.

tionship (OR 0.08, CI 0.01-0.70, $p=0.02$ and OR 0.12, CI $0.02-0.63, p=0.01$ ) for villous trophoblasts and stroma, respectively (Fig. 3).

\section{DISCUSSION}

Higher concentrations of inflammatory cytokines have been documented in amniotic fluid, cord blood, and tracheal aspirates of premature infants who subsequently developed BPD (12-17), whereas anti-inflammatory cytokines were found to be absent or in lower concentrations in bronchoalveolar lavage fluid of premature infants with BPD $(17,18)$. These patterns may depend upon the expression of pro- and anti-inflammatory cytokines in multiple placental compartments. The results of this study support the hypothesis that placental tissues of infants who developed BPD manifest down-regulation of IL-10 expression, while robustly expressing IL-6.

Our original hypothesis proposed an imbalance of cytokines among infants who develop BPD with an increase in proinflammatory cytokines and a decrease in anti-inflammatory cytokines in the placenta. Postulating a predominance of placental IL-6 staining relative to IL-10 is based upon the observations that IL-6 is elevated in amniotic fluid, cord blood, and tracheal aspirates of premature infants who subsequently develop BPD (12-18). Furthermore, it is recognized that cytokines can be secreted from cells in the decidua and chorion, as well as from infiltrating macrophages $(20,21)$. One mechanism responsible for the activation of an inflammatory response in the premature/preterm lungs may be direct damage, as ventilator-induced injury can induce mRNA expression of IL-6 in the premature/preterm lungs (22). Inhalation of amniotic fluid by the fetus may favor the extension of the placental/amniotic fluid environment into the tracheobronchial tree, thus promoting inflammation, as proposed by Ghezzi et al., (14). Alternatively, placental expression of proinflammatory mediators may prompt a fetal systemic inflammatory response with increased endothelial permeability, capillary leakage, and diffuse alveolar damage predisposing to BPD. Increased cord blood concentration of IL-6 among infants destined to develop BPD is supportive of this notion (12). The results of this study, in contrast, did not demonstrate differences in IL-6 staining in any of the placental compartments of infants with BPD compared with those without BPD. Similar expression of IL-6 in placental tissue with differences in IL-10 expression would certainly not preclude more prominent IL-6 production in other tissues and body fluid in response to inflammation and lung injury. We propose that in the absence of placental IL-10 in BPD cases, the inflammatory function of IL-6 remains unchecked.

It is interesting that we did not see a difference in the incidence of histologic chorioamnionitis between newborns with and without BPD. Although some studies have found a positive association between chorioamnionitis and $\operatorname{BPD}(8,9)$, other studies have not supported the notion of chorioamnionitis as a risk factor for BPD $(23,24)$. This conflicting data likely represents differences in definitions of chorioamnionitis and BPD, time periods studied, and antenatal factors, such as maternal steroid use. Chorioamnionitis is characterized by higher amniotic fluid and cord blood concentrations of IL-6 $(25,26)$; however, no studies specifically examined the relationship between chorioamnionitis, as defined by neutrophil response, and placental cytokine expression. The relationship between the BPD pathology and cytokine response is likely more complex than we understand.

The results of this study support a critical anti-inflammatory and immunomodulatory role of IL-10 even before birth in the development of BPD. Recent reports have suggested that IL-10 placental expression is GA dependent (27), and we did not see prominent IL-10 staining in the cord, membranes, and placenta. However, despite this, the presence of placental IL-10 in the villous trophoblast was associated with a reduction in the odds of developing BPD. This relationship was present in unadjusted and adjusted analyses for differences in GA and race. In agreement with our findings is the observation that the absence of IL-10 in tracheal aspirates in infants $<27$ wk is associated with a higher risk of developing BPD (16). Similarly, poor IL-10 expression has been reported in preterm infants with hyaline membrane disease $(18,28)$. Although these latter studies did not specifically look at chronic lung disease, it may be that persistent and unchecked inflammation plays a role in the evolution of BPD.

Recent work has shown genetic heritability to $\operatorname{BPD}(8,9)$. Emerging data suggests that genetic variations in IL-10 gene regulation may ultimately affect the anti-inflammatory response. IL-10 regulation is complex and it is subject to genetic influence $(29,30)$. The single-nucleotide polymorphism (SNP) at $-1082 \mathrm{G}$ allele has been associated with a higher IL-10 expression in certain cell types (31). Moreover, recent work has shown that preterm infants who carry two $G$ alleles of the IL-10 (-1082) SNP were at a prominently reduced risk for neonatal cerebral, eye, and lung (specifically BPD) damage (32). Although it is plausible that polymorphic changes in the 
IL-10 promoter may be differentially used in different cell types in response to inflammatory triggers, our data suggest that IL-10 expression in the villous tissue is not uniquely influenced by chorioamnionitis. We propose that IL-10 downregulation in the placenta of infants with BPD involves additional events, such as an excessive production of prostanoids or dysregulation of the hormonal cascade.

There are important limitations to this study that should be acknowledged. Although we matched for variables (sex, period of birth, and BW) that previously have shown to affect BPD (33-35), there may be unidentified variables influencing the results of a case-control retrospective study. Although we felt matching for the above variables was clinically relevant, we acknowledge potential bias in case selection in a retrospective analysis. The cohort of this study represents $\sim 46 \%$ and $23 \%$ of BPD and no BPD infants, respectively, available from our NICU. Despite our matching of the above variables, there were group differences in GA and trends in differences in race. Racial differences in the development of BPD have been previously reported and matching on race would have made it impossible to attain a reasonable sample size (35). The lower $\mathrm{GA}$ and race were adjusted for in the logistic regressions. We also are aware that there are other criteria being used by some to define BPD, such as an oxygen challenge reduction test (36). This methodology was not being performed at the time period of our study and our definition of oxygen use at 36-wk PMA is within the scope of the National Institutes of Health consensus conference definition of BPD (37).

We also recognize the variability of immunohistochemical staining and the subjectivity of grading. To allow for a more accurate statistical analysis, each batch of staining consisted of patients with BPD and their matched controls, in addition to a standard reference control. The scoring system used for determining the extent of staining was qualitative rather than quantitative; ideally, a system that determines the extent of cytokine staining both in terms of placental area involved and degree of staining would be desirable. Despite this, the analysis of interobserver reliability was reassuring with a kappa value of 0.82 . In addition, the categorical analysis of our data as either "no staining" or "any staining" also decreased subjectivity.

Our findings support a "cytokine balance" theory. Logistic regression analysis shows an independent association between IL-10 and BPD. This suggests that baseline inflammation is present, but the absence of anti-inflammatory mediators in the placenta may initiate fetal inflammatory responses. We believe that this study is important as it presents a prenatal or antenatal environment that may ultimately affect the postnatal course of an infant. Further studies of placental examination may allow us to better identify those premature infants at increased risk for particular morbidities.

Acknowledgments. We thank Richard Tucker for his help with statistical analysis and Paul Monfils of the Rhode Island Hospital Core Research Laboratory for his help with immunohistochemical analysis.

\section{REFERENCES}

1. Klinger G, Sirota L, Lusky A, Reichman B 2006 Bronchopulmonary dysplasia in very low birth weight infants is associated with prolonged hospital stay. J Perinatol 26:640-644

2. Furman L, Baley J, Borawski-Clark E, Aucott S, Hack M 1996 Hospitalization as a measure of morbidity among very low birth weight infants with chronic lung disease. J Pediatr 128:447-452

3. Short EJ, Klein NK, Lewis BA, Fulton S, Eisengart S, Kercsmar C, Baley J, Singer LT 2003 Cognitive and academic consequences of bronchopulmonary dysplasia and very low birth weight: 8 year outcomes. Pediatrics 112:e359

4. Lewis BA, Singer LT, Fulton S, Salvator A, Short EJ, Klein N, Baley J 2002 Speech and language outcomes of children with bronchopulmonary dysplasia. J Commun Disord 35:393-406

5. Fanaroff AA, Stoll BJ, Wright LL, Carlo WA, Ehrenkranz RA, Stark AR, Bauer CR, Donovan EF, Korones SB, Laptook AR, Lemons JA, Oh W, Papile LA, Shankaran S, Stevenson DK, Tyson JE, Poole WK; NICHD Neonatal Research Network 2007 Trends in neonatal morbidity and mortality for very low birthweight infants. Am J Obstet Gynecol 196:147.e1-147.e8

6. O'Brodovich HM, Mellins R 1985 Bronchopulmonary dysplasia. Unresolved neonatal acute lung injury. Am Rev Respir Dis 132:694-709

7. Coalson JJ 2006 Pathology of bronchopulmonary dysplasia. Semin Perinatol 30:179-184

8. Bhandari V, Bizzarro MJ, Shetty A, Zhong X, Page GP, Zhang H, Ment LR, Gruen JR; Neonatal Genetics Study Group 2006 Familial and genetic susceptibility to major neonatal morbidities in preterm twins. Pediatrics 117:1901-1906

9. Lavoie PM, Pham C, Jang KL 2008 Heritability of bronchopulmonary dysplasia, defined according to the consensus statement of the national institutes of health Pediatrics 122:479-485

10. Watterberg KL, Demers LM, Scott SM, Murphy S 1996 Chorioamnionitis and early lung inflammation in infants in whom bronchopulmonary dysplasia develops. Pediatrics 97:210-215

11. Viscardi RM, Muhumuza CK, Rodriguez A, Fairchild KD, Sun CC, Gross GW, Campbell AB, Wilson PD, Hester L, Hasday JD 2004 Inflammatory markers in intrauterine and fetal blood and cerebrospinal fluid compartments are associated with adverse pulmonary and neurologic outcomes in preterm infants. Pediatr Res 55:1009-1017

12. Yoon BH, Romero R, Kim KS, Park JS, Ki SH, Kim B, Jun JK 1999 A systemic fetal inflammatory response and the development of bronchopulmonary dysplasia. Am J Obstet Gynecol 181:773-779

13. Yoon BH, Romero R, Jun JK, Park JK, Park JD, Gezzi F, Kim B 1997 Amniotic fluid cytokines (interleukin-6, tumor necrosis factor-alpha, interleukin-1 beta, and interleukin-8) and the risk for the development of bronchopulmonary dysplasia. Am J Obstet Gynecol 177:825-830

14. Ghezzi F, Gomez R, Romero R, Yoon BH, Edwin SS, David C, Janisse J, Mazor M 1998 Elevated interleukin-8 concentrations in amniotic fluid of mothers whose neonates subsequently develop bronchopulmonary dysplasia. Eur J Obstet Gynecol Reprod Biol 78:5-10

15. Bagchi A, Viscardi RM, Taciak V, Ensor JE, McCrea KA, Hasday JD 1994 Increased activity of interleukin- 6 but not tumor necrosis factor- $\alpha$ in lung lavage of premature infants is associated with the development of bronchopulmonary dysplasia. Pediatr Res 36:244-252

16. Garingo A, Tesoriero L, Cayabyab R, Durand M, Blahnick M, Sardesai S, Ramanathan R, Jones C, Kwong K, Li C, Minoo P 2007 Constitutive IL-10 expression by lung inflammatory cells and risk for bronchopulmonary dysplasia. Pediatr Res 61:197-202

17. Oei J, Lui K, Wang H, Henry R 2002 Decreased interleukin-10 in tracheal aspirates from preterm infants developing chronic lung disease. Acta Paediatr 91:1194-1199

18. Jones CA, Cayabyab RG, Kwong YC, Scotts C, Wong B, Hamden H, Minoo P, deLemos RA 1996 Undetectable interleukin-10 and persistent interleukin- expression early in hyaline membrane disease: a possible developmental basis for the predisposition to chronic lung inflammation in preterm newborns. Pediatr Res 39:966-975

19. Shennan AT, Dunn MS, Ohlsson A, Lennox K, Hoskins EM 1988 Abnormal pulmonary outcomes in premature infants: prediction from oxygen requirement in the neonatal period. Pediatrics 82:527-532

20. Berner R, Niemeyer CM, Leititis JU, Funke A, Schwab C, Rau U, Richter K, Tawfeek MS, Clad A, Brandis M 1998 Plasma levels and gene expression of granulocyte colony-stimulating factor, tumor necrosis factor-alpha, interleukin (IL)1 beta, IL-6, IL-8, and soluble intercellular adhesion molecule-1 in neonatal early onset sepsis. Pediatr Res 44:469-477

21. Dudley DJ, Collmer D, Mitchell D, Trautman MS 1996 Inflammatory cytokine mRNA in human gestational tissues: implications for term and preterm labor. J Soc Gynecol Investig 3:328-335

22. Naik AS, Kallapur SG, Bachurski CJ, Jobe AH, Michna J, Kramer BW, Ikegami M 2001 Effects of ventilation with different positive end-expiratory pressures on cytokine expression in the preterm lamb lung. Am J Respir Crit Care Med 164:494498

23. Redline RW, Wilson-Costello D, Hack M 2002 Placental and other perinatal risk factors for chronic lung disease in very low birth weight infants. Pediatr Res 52:713-719

24. Kent A, Dahlstrom JE 2004 Chorioamnionitis/funisitis and the development of bronchopulmonary dysplasia. J Paediatr Child Health 40:356-359

25. Yoon BH, Romero R, Kim CJ, Jun JK, Gomez R, Choi JH, Syn HC 1995 Amniotic fluid interleukin 6: a sensitive test for antenatal diagnosis of acute inflammatory 
lesions of preterm placenta and prediction of perinatal morbidity. Am J Obstet Gynecol 172:960-970

26. Pacora P, Chaiworapongsa T, Maymon E, Kim YM, Gomez R, Yoon BH, Ghezzi F, Berry SM, Qureshi SM, Kim JC, Nadar N, Romero R 2002 Funisitis and chorionic vasculitis: the histological counterpart of the fetal inflammatory response syndrome. J Matern Fetal Neonatal Med 11:18-25

27. Hanna N, Hanna I, Hleb M, Wagner E, Dougherty J, Balkundi D, Padbury J, Sharma S 2000 Gestational age dependent expression of IL-10 and its receptor in human placental tissue and isolated cytotrophoblasts. J Immunol 164:5721-5728

28. Kwong KY, Jones CA, Cayabyab R, Lecart C, Khuu N, Rhandhawa I, Hanley JM, Ramanatha R, deLemos RA, Minoo R 1998 The effects of IL-10 on proinflammatory cytokine expression (IL-1beta and IL-8) in hyaline membrane disease. Clin Immunol Immunopathol 88:105-113

29. Westendorp RG, Langermans JA, Huizinga TW, Elouali AH, Verweij CL, Boomsma DI, Vandenbrouke JP 1997 Genetic influence on cytokine production and fatal meningococcal disease. Lancet 349:170-173

30. Turner DM, Williams DM, Sankaran D, Lazarus M, Sinnott PJ, Hutchinson IV 1997 An investigation of polymorphisms in the interleukin-10 gene promoter. Eur J Immunogenet $24: 1-8$

31. Schaaf BM, Boehmke F, Esnaashari H, Seitzer U, Kothe H, Maass M, Zabel P, Dalhoff K 2003 Pneumococcal septic shock is associated with the interleukin-101082 gene promoter polymorphism. Am J Respir Crit Care Med 168:476-480
32. Dordelmann M, Kerk J, Dressler F, Brinkhaus MJ, Bartels DB, Dammann CE, Dork T, Dammann O 2006 Interleukin-10 high producer allele and ultrasound defined periventricular white matter abnormalities in preterm infants: a preliminary study. Neuropediatrics 37:130-136

33. Oh W, Poindexter BB, Perritt R, Lemons JA, Bauer CR, Ehrenkranz RA, Stoll BJ, Poole K, Wright LL; Neonatal Research Network 2005 Association between fluid intake and weight loss during the first ten days of life and risk of bronchopulmonary dysplasia in extremely low birthweight infants. J Pediatr 147:786-790

34. Costeloe K, Hennessy E, Gibson AT, Marlow N, Wilkinson AR 2000 The EPICure study: outcomes to discharge from hospital for infants born at the threshold of viability. Pediatrics 106:659-671

35. Ehrenkranz RA, Walsh MC, Vohr BR, Jobe AH, Wright LL, Fanaroff AA, Wrage LA, Poole K; National Institute of Child Health and Human Development Neonatal Research Network 2005 Validation of the National Institutes of Health consensus definition of bronchopulmonary dysplasia. Pediatrics 116:1353-1360

36. Walsh MC, Yao Q, Gettner P, Hale E, Collins M, Hensman A, Everette R, Peters N, Miller N, Muran G, Auten K, Newman N, Rowan G, Grisby C, Arnell K, Miller L, Ball B, McDavid G; National Institute of Child Health and Human Development Research Network 2004 Impact of a physiologic definition on bronchopulmonary dysplasia rates. Pediatrics 114:1305-1311

37. Jobe AH, Bancalari E 2001 Bronchopulmonary dysplasia. Am J Respir Crit Care Med 163:1723-1729 